\title{
DNA Nanotechnology
}

\author{
Seung-Hyun Lee and Chengde Mao \\ Department of Chemistry, Purdue University, West Lafayette, IN, USA
}

There has been tremendous interest in recent years in developing concepts and approaches for structural control and mechanical devices at the nanometer scale. While significant work continues, an important advance is the realization that the molecular recognition properties of biological macromolecules could be used to form complex networks of potentially useful species for optical, electronic, and sensing applications $(1,2)$.

DNA molecules today have been employed as chemical building blocks to build nanometer-sized structures. Pioneering research by Seeman and his coworkers has laid the foundation for construction of nanoscale structures from DNA, based on its physical properties. DNA has several structural considerations that make it well-suited for making nanostructures-its complementary strands, an antiparallel double-helical backbone that is largely regular regardless of sequence, a persistence length (a measure of "stiffness") around $50 \mathrm{~nm}$, and a design that makes it possible to read its sequence from the outside, even when the strands are paired (1). The recognition of DNA strands by their complements can be used for more than the formation of a simple double helix. Biologists recognized in the early 1970s that single-stranded overhangs (sticky ends) could be used to direct intermolecular association of different DNA molecules (1). The intermolecular structures formed are predictable, due to the base pairing rules. Such sticky ends (usually four to nine bases long in DNA nanotechnology) are, thus, convenient in constructing intermolecular structures, especially because complementarity is easy to program. However, a DNA double helix is just a linear molecule. Formation of complicated structures requires branched DNA molecules. Such molecules are found in biological systems as the ephemeral four-arm Holliday junction, an intermediate in genetic recombination (1). The basic notion of structural DNA nanotechnology is to combine stable branched DNA molecules with sticky-ended cohesion or other forms of cohesion that are structurally well-defined.

Here we examine some recent developments and achievements in DNA nanotechnology in the authors' group. These developments may be divided into three categories, the use of single-stranded DNA to prepare periodic arrays, the design of DNA motors, and DNA-based nanofabrication.

\section{DNA Construction}

The formation and assembly of molecular-scale building blocks into well-defined structures is the essence of nanotechnology. Recently, we were able to construct a nanoscale geometric structure, DNA triangles, based on a tensegrity strategy (3). Instead of joining rigid building blocks, we have used rigid struts and flexible tendons to build nanometer-scale equilateral triangles (4). Each individual four-arm junction has four helical arms, which stack into two pseudo-continuing DNA duplexes. Each pseudoDNA duplex is rigid, but the interhelical angle, the angle between the two pseudo-DNA duplexes, is very flexible. How to overcome the flexibility presents a great chal- lenge for structural control. We have developed a strategy to build rigid structures from these flexible components (Figure 1). Three four-arm junctions are joined together in

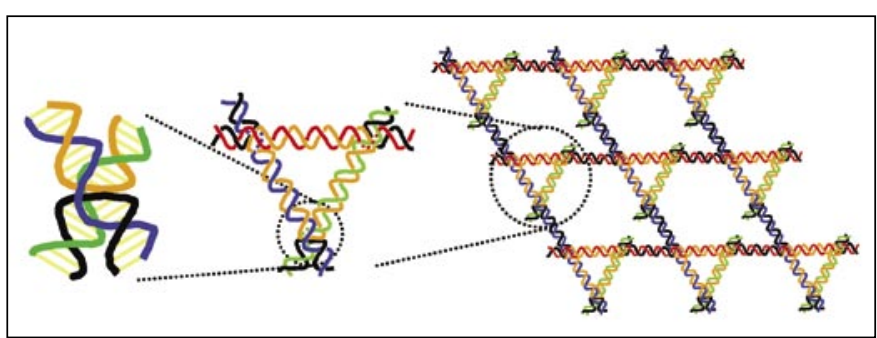

Figure 1. The design and 2-dimensional self-assembly of DNA triangles.

a DNA nanotriangle. Each vertex of the triangle is made of a four-arm junction, and each side of the triangle is a DNA duplex. In the triangular design, the crossovers in the four-arm junctions are used as tendons to join the rigid struts. The resulting triangles can further self-assemble into 1- and 2-dimensional arrays when proper sticky ends are added. All these structures have been directly visualized by atomic force microscopy (AFM). The tensegrity strategy may become an important strategy for designing biomimetic nanomaterials.

\section{DNA Nanomachinery: An Autonomous DNA Nanomotor and Its Brake}

DNA can be also used to build nanoscale machines, and a number of DNA-based nanomechanical devices have been already made. However, most of these nanomotors are operated by cyclic changes of the chemical environment. In contrast, cellular protein motors constantly extract chemical energy from chemical bonds and convert this energy to produce mechanical motion. It will be of great interest to design a synthetic nanomotor that cycles freely under continuous stimulation, just as natural protein motors do. Recently, we have introduced a novel DNA nanomotor that has an autonomous feature (5). The motor is powered by a 10-23 RNAcleaving DNA enzyme (6), which was discovered through in vitro selection. The 10-23 enzyme cleaves its RNA substrate into two short fragments and then releases the pieces. This process causes the DNA molecule to change its conformation and results in nanoscale motions (Figure 2 ).

The 10-23 DNA enzyme consists of a 15-base catalytic core and two flanking 7-base-long substrate-recognition arms. The DNA enzyme binds to its RNA substrate through Watson-Crick base pairing and cleaves the RNA into two short fragments. When the DNA enzyme is not bound to its substrate, divalent cations cause the single-stranded DNA enzyme to collapse into a closed coil as a result of entropic forces. Under these conditions, the overall conformation of the DNA "motor" is quite compact. When the DNA enzyme binds to its RNA substrate, the single-stranded 


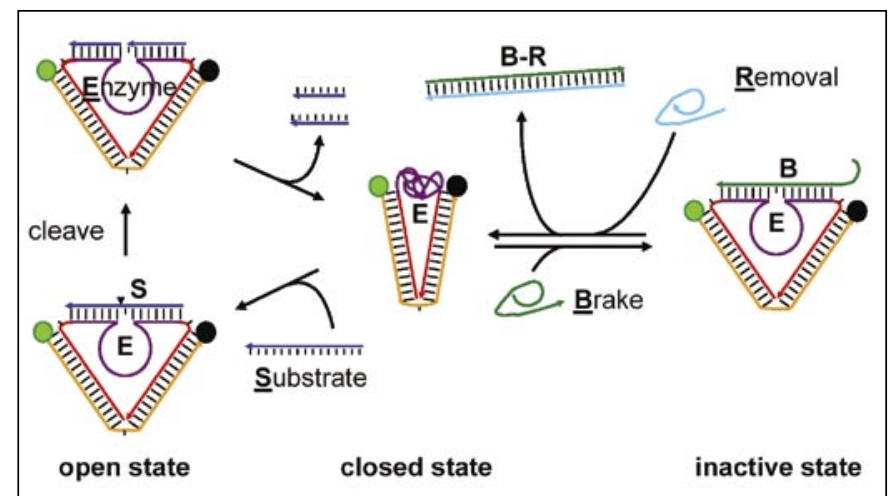

Figure 2. Structure and operation of an autonomous DNA nanomotor. The RNA-cleaving 10-23 DNA enzyme domain is colored dark purple. Solid green and black circles represent rhodamine green and black hole quencher-1, respectively. Except that strand $S$ is an RNA molecule, all other strands are DNA. The arrowhead above the $S$ strand indicates the cleavage site. Reproduced with permission from Reference 7. Copyright 2004. Am. Chem. Soc.

DNA enzyme forms a bulged duplex with the substrate. This bulged duplex pushes the two helical domains of the DNA motor apart and leads the motor to adopt an open state; upon binding, the DNA enzyme cleaves its substrate into two short fragments. The resulting fragments have a lower affinity for the DNA enzyme than the intact substrate and therefore, dissociate from the DNA motor. Consequently, the DNA motor returns to the closed state and can undergo the next cycle of substrate binding, cleavage and dissociation, or close/open motion.

Controlling nanoscale motions is also an important aspect in constructing better nanodevices. For that purpose, we have incorporated a reversible brake into this DNA motor (7). An uncleavable DNA analog of the RNA substrate has been chosen as the brake. Like the RNA substrate, the brake strand can bind to the enzyme, but the brake strand is engineered to form a longer bulged duplex than an RNA substrate with the enzyme. The enzyme will prefer binding a brake to binding the RNA substrate because of an affinity difference. After binding with a brake strand, the DNA motor will stop moving. To restart the motor, the brake strand has to be removed, which is accomplished by a strand displacement mechanism. The brake strand has a 10-base-long tail that does not involve its inhibition function but rather its removal. A brake removal strand is fully complementary to the brake strand, and they form a long duplex. The removal strand first base pairs with the brake strand at the tail region and then pulls the brake strand out of the motor through branch migration. When the brake strand is removed from a motor, the enzyme becomes active, binds a target RNA, and the motor starts to move again. The addition and removal of a brake to and from a DNA motor are reversible and can be repeated.

The motion and brake effect of the DNA motor have been demonstrated by gel electrophoresis and fluorescence resonance energy transfer (FRET) techniques. This work shows that combining proper design with DNA catalytic function can open doors to new opportunities in construction of dynamic nanomaterials, nanorobotics, and nanodevices.

\section{Nanofabrication with DNA Nanostructures}

There have been reports on using DNA to fabricate nanowires. However, the current methods are limited to fabricating only linear structures. For practical applications, it is important to have an ability to build more complex structures. Since it has become relatively easy to construct complex DNA structures through bottom-up selfassembly of designed DNA motifs, it is possible to produce well-defined nanopatterns with designed DNA structures serving as effective masks in direct replication.

We have recently demonstrated the fabrication of 1- and 2-dimensional metallic nanopatterns down to several nanometers (8). Figure 3 illustrates the process. First, DNA

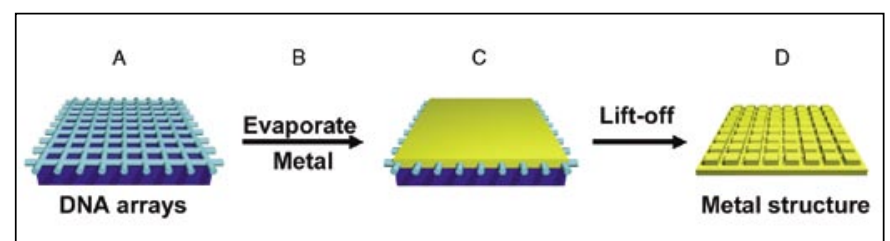

Figure 3. DNA-based nanofabrication. (A) DNA nanostructures are self-assembled in solution and deposited on mica. (B and C) Metal evaporates until a continuous metal film forms and covers the DNA mask on the mica surface. (D) The metal film, after lifted off from the DNA-mica, exhibits a negative replica of the DNA mask. Reproduced with permission from Reference 8. Copyright 2004. Wiley-VCH.

arrays were assembled and deposited onto a flat substrate. A thin metal film was then vapor-deposited onto the substrate. After being mechanically separated from the substrate, the metallic film contained a negative replica of the DNA nanostructures. The experimental results have demonstrated that this method can fabricate complex surface patterns with well-defined structures and periodicity. This novel fabrication method might be used in many different applications, such as nanoscale electronic circuits, miniaturized sensors, nanofluidcs and super high-resolution display with nanoscale pixels.

\section{Future Challenges}

One key task in DNA nanotechnology and its practical applications is extension of the array-making capability from two dimensions to three dimensions. In addition, heterogonous molecules must be incorporated into DNA arrays, so that the goals of ordering biological macromolecules for diffraction study and of organizing nanoelectronic circuits can be achieved $(9,10)$. It is a major problem to crystallize biological macromolecules for structural study. A constructed periodic DNA lattice (a crystal) could be used as a "host" to order "guest" biological macromolecules inside the lattice (11). The conjugated lattice can diffract X-rays and thereby enable structure determination. The same method could be used to position and orient components of molecular electronic devices with nanometer-scale precision (12-14). The DNA structures can also be used as templates or masks for nanofabrications $(8,15)$. Collectively, these methods will make it possible to fabricate nanostructures with technologically useful materials. Such ability is the key to future computers, catalysis, solar energy utilization, and hydrogen production and storage.

DNA nanomachines are promising tools in nanorobotics, nanofacturing, computation, and healthcare. For example, it is conceivable to build tiny machines to detect diseases at very early stages, to autonomously administer medicines (16), and to perform surgeries. 
DNA nanotechnology is still in its infancy. However, the future of this field is very promising, with many new concepts and novel strategies developed in recent years. For example, a strategy has been developed to clone geometric DNA nanostructures (17), and a DNA walking device has been constructed (18). In the next decade or so, we believe, DNA nanotechnology will start to generate practical technologies for many applications, such as protein structure determination for biomedical research, sensitive detectors for environmental protection and disease diagnosis, and high-density arrays for information storage. DNA, long known as a blueprint that makes each species what it is, may soon be used as both template and building block for the inventions of humans.

\section{Acknowledgments}

The authors would like to thank members of the Mao group at Purdue University for helpful discussion.

\section{References}

1.Seeman, N.C. 2003. At the crossroads of chemistry, biology, and materials: structural DNA nanotechnology. Chem. Biol. 10:1151-1159.

2.Mirkin, C.A. and J.J. Storhoff. 1999. Programmed materials synthesis with DNA. Chem. Rev. 99:1849-1862.

3.Pugh, A. 1976. An Introduction to Tensegrity. University of California Press, Berkeley, CA.

4.Liu, D., M. Wang, Z. Deng, R. Walulu, and C. Mao. 2004. Tensegrity: construction of rigid DNA triangles with flexible four-arm DNA junctions. J. Am. Chem. Soc. 126:2324-2325.

5.Chen, Y., M. Wang, and C. Mao. 2004. An autonomous DNA nanomotor powered by a DNA enzyme. Angew. Chem Int. Ed. 43:3554-3557.

6.Santoro, S.W. and G.F. Joyce. 1997. A general purpose RNA-cleaving DNA enzyme. Proc. Natl. Acad. Sci. USA 94:4262-4266.

7.Chen, Y. and C. Mao. 2004. Putting a brake on an autonomous DNA nanomotor. J. Am. Chem. Soc. 126:8626-8627.

8.Deng, Z. and C. Mao. 2004. Molecular lithography with DNA nanostructures. Angew. Chem. Int. Ed. 43:4068-4070.

9.Seeman, N.C. 2003. DNA in a material world. Nature 421:427-431.

10.Seeman, N.C. 2003. DNA nanotechnology. Materials Today Jan:24-29.

11.Yan, H., S.H. Park, G. Finkelstein, J.H. Reif, and T.H. LaBean. 2003. DNA-templated self-assembly of protein arrays and highly conductive nanowires. Science 301:1882-1884.

12.Keren, K., R.S. Berman, E. Buchstab, U. Sivan, and E. Braun. 2003. DNA-templated carbon nanotube field-effect transistor. Science 302:1380-1382.

13.Mirkin, C.A., R.L. Letsinger, R.C. Mucic, and J.J. Storhoff. 1996. A DNA-based method for rationally assembling nanoparticles into macroscopic materials. Nature 382:607-609.

14.Alivisatos, A.P., K.P. Johnsson, X.G. Peng, T.E. Wilson, C.J. Loweth, M.P. Bruchez, and P.G. Schultz. 1996. Organization of 'nanocrystal molecules' using DNA. Nature 382:609-611.

15.Liu, D., S.H. Park, J.H. Reif, and T.H. LaBean. 2004. DNA nanotubes self-assembled from triple-crossover tiles as templates for conductive nanowires. Proc. Natl. Acad. Sci. USA 101:717-722.

16.Benenson, Y., B. Gil, U. Ben-Dor, R. Adar, and E. Shapiro. 2004. An autonomous molecular computer for logic control of gene expression. Nature 429:423-429.

17.Shih, W.M., J.D. Quispe, and G.F. Joyce. 2004. A 1.7-kilobase singlestranded DNA that folds into a nanoscale octahedron. Nature 427:618621.

18.Sherman, W.B. and N.C. Seeman. 2004. A precisely controlled DNA biped walking device. Nano Lett. 4:1203-1207. 